\title{
Treinamento funcional: contribuições para a qualidade de vida de moradores da área rural de Paripiranga/BA
}

\author{
Treinamento funcional: contribuições para a qualidade de vida \\ dos moradores da área rural de Paripiranga / BA
}

\author{
Laine Santos da Silva ${ }^{1}$ \\ Davi Soares Santos Ribeiro ${ }^{1}$
}

\section{RESUMO}

0 Treinamento Funcional é considerado como um conjunto de exercícios direcionados para trabalhar a funcionalidade do corpo e suas capacidades físicas. Sua principal característica está ligada a um conjunto de exercícios pensados e preparados para trabalhar o corpo sob diferentes vertentes e estímulos, buscando, assim, alcançar padrões de movimentos cada vez mais eficientes. Nesse sentido, o estudo tem como objetivo analisar os benefícios proporcionados pelo treinamento funcional para a promoção de qualidade de vida em moradores da área rural de Paripiranga (BA). 0 estudo caracteriza-se como uma pesquisa descritiva com abordagem qualitativa. Para a coleta dos dados, utilizou-se questionários IPAQ e o WHOQOL-bref, para análise do nível de atividade física e qualidade de vida, respectivamente. A amostra foi constituída por 30 participantes, de ambos os sexos, sendo 15 participantes ativos praticantes de treinamento funcional e 15 participantes considerados insuficientemente ativos. Os resultados revelaram que os indivíduos considerados ativos apresentaram aumento dosíndices das variáveis ligadas às melhorias da qualidade de vida, tais como: melhor disposição para o sono e repouso; maior mobilidade; e maior disposição para o desenvolvimento das atividades da vida cotidiana quandocomparados aos dados obtidos pelos participantes insuficientemente ativos. Conclui-se que a prática regular de um programa de treinamento funcional pode contribuir satisfatoriamente para o aumento das variáveis relacionadas à qualidade de vida.

\section{PALAVRAS-CHAVE}

Treinamento Funcional; Qualidade de Vida; Exercício Físico.

\footnotetext{
${ }^{1}$ UniAGES Centro Universitário, Paripiranga/BA.
} 


\section{ABSTRACT}

Functional Training is considered as a set of exercises aimed at working the functionality of the body and its physical capabilities. Its main feature is linked to a set of exercises designed and prepared to work the body under different strands and stimuli, seeking to achieve increasingly efficient patterns of movement. In this sense, the objective of this study is to analyze the benefits provided by functional training for the promotion of quality of life in rural residents of the city of Paripiranga (BA). The study is characterized as a descriptive research with a qualitative approach. To collect the data, IPAQ and WHOQOL-bref questionnaires were used to analyze the level of physical activity and quality of life, respectively. The sample consisted of 30 participants, of both sexes, 15 active participants practicing functional training and 15 participants considered insufficiently active. The results showed that the individuals considered active had an increase in the indices of variables related to improvements in quality of life, such as: better disposition for sleep and rest; greater mobility; and greater willingness to develop the activities of daily life when compared to the data obtained by the insufficiently active participants. It is concluded that the regular practice of a functional training program can contribute satisfactorily to the increase of variables related to quality of life.

\section{KEYWORDS}

Functional Training; Quality of life; Physical exercise. 


\section{INTRODUÇÃO}

0 treinamento funcional ( $\mathrm{T}$ F) refere-se a um conjunto de exercícios praticados como a finalidade do preparo físico e/ou aprimoramento de habilidades, cuja execução está relacionada aexecução de ações motoras realizadas no cotidiano (MICHAELIS, 2009). Segundo Gelatti (2009), o treinamento funcional define-se como um novo conceito de treinamento especializado que utiliza o próprio corpo como instrumento de trabalho com a finalidade de realizar movimentos de forma integrada e eficiente.

OTF possui uma abordagem dinâmica, desafiadora, complexae,principalmente motivante, pois é construído tendo como base movimentos do cotidiano ou movimentos específicos esportivos a fim de treinar o indivíduo a partir da funcionalidade dos movimentos (RIBEIRO, 2006). Sua principal característica está ligada a um conjunto de exercícios para trabalhar o corpo sob diferentes vertentes e estímulos, buscando, assim, alcançar padrões de movimentos cada vez mais eficientes como empurrar, puxar, agachar, girar, lançar, dentre outros.

Conforme Campos e Coraucci Neto (2008), a essência do TF está baseada na melhoria dos aspectos neurológicos, através de exercícios que desafiam os diversos componentes do sistema nervoso e resultando em melhorias nas tarefas do dia a dia, bem como nos gestosesportivos.OTFérealizadocontraumaresistência, em que a força gerada beneficia diretamente a execução de atividades da vida diária e movimentos associados ao esporte (ACM, 2009).

O seu surgimento está associado ao reconhecimento conquistado pela contribuição de seus exercícios, associados aos trabalhos das especialidades na reabilitação de lesões de soldados na Segunda Guerra Mundial e em atletas olímpicos nos anos 1950, os quais demonstraram uma melhora em suas capacidades físicas (BOSSI, 2011). No Brasil, o TF foi desenvolvido pelo professor Luciano D’Elia, no final da década de 1990, trazend o o treinamento funcional como sendo um conjunto deatividades que auxiliam no desenvolvimento dos esportes voltados para as lutas. E, logo em seguida foi se expandindo para as demais praticantes de atividades físicas que manifestaram interesse por mais esse novo tipo de treinamento (FARIAS, 2015).
0 treinamento funcional foi ganhando reconhecimento perante os programas já existentes, por se tratar de uma nova metodologia que busca trabalhar o corpo em sua totalidade, além de proporcionar inúmeros benefícios para todos os seus sistemas e influenciar nas suas atividades diárias, visto que esse método consegue atender tanto o indivíduo mais condicionado como o menos condicionado, criando, assim, um ambiente dinâmico de treino (GOLDENBERG; TWIST, 2002).

Partindo desse princípio, o estudo tem como objetivo analisar os benefícios proporcionados pelo treinamento funcional para a promoção de qualidade de vida em moradores da área rural de Paripiranga (BA).

Segundo Campos e Coraucci Neto (2008), a essência do treinamento funcional está baseada na melhoria dos aspectos neurológicos, através de exercícios que desafiam os diversos componentes do sistema nervoso e resultando em melhorias nas tarefas do dia a dia, bem como nos gestos esportivos.

Para Silva (2011), deve-se planejar e programar tais exercícios atendendo ao nível de carga (externa-interna) em relação ao nível de rendimento do aluno e ao processo global de treinamento. Recomenda-se refletir como deve ser direcionado o foco do treinamento, uma vez que os avanços da sociedade moderna tendem a conduzir o indivíduo ao sedentarismo e à hipocinesia, levando as pessoas a movimentos cada vez mais curtos, menos variados e menos frequentes.

De acordo com Campos e Coraucci Neto (2008), o treinamento funcional, bem como a escolha de seus exercícios deve estimular o corpo humano de maneira a adaptá-lo para as atividades normais da vida cotidiana. Um aspecto essencial neste tipo de treinamento está ligado à necessidade de se pensar a prática de exercícios que estimulem a propriocepção, a força, a resistência muscular, a flexibilidade, a coordenação motora, o equilíbrio e o condicionamento físico.

Segundo Silva (2011), para um treinamento ser funcional devem-se cumprir todos os critérios determinados para cada estímulo promovido, levando em consideração que o processo de periodização estabelecido deve priorizar o controle e manipulação das variáveis existentes de forma que se concretize em uma prescrição que contenha a dose adequada de exercício que o indivíduo deve realizar na unidade de treinamento. Destaca-se que, se treinamento for 
realizado adequadamente, consequentemente, o estado psicobiológico do praticante será adequadamente estimulado, gerando respostas e adaptações positivas.

Da mesma forma, esse tipo de treinamento está fundamentado em duas diretrizes, que são: o respeito à individualidade biológica, que é o primeiro passo para a prescrição de um programa de exercícios seguros e coerentes; eaoprincípiodaespecificidadedotreinamento, na qual as adaptações que ocorrem no corpo humano, decorrente dos estímulos proporcionados pelo exercício e determinante de cada programa de treinamento (CAMPOS; CORAUCCI NETO, 2008).

0 treinamento funcional tem como base uma prescrição coerente e segura de exercícios que permitam a estimulação do corpo humano de maneira capaz de evoluir todas as qualidades do sistema musculoesquelético como velocidade, força, equilíbrio, coordenação, flexibilidade, lateralidade, resistência cárdio e neuromuscular e, também, motivação através da manutenção do centro de gravidade do corpo (CAMPOS; CORAUCCI NETO, 2008).

Partindo por esse princípio, torna-se importante considerar que a construção do planejamento e prescrição do treinamento funcional devem considerar todos os princípios biológicos de cada indivíduo para, assim, determinar quais capacidades poderão ser priorizadas diante do objetivo esperado por seus praticantes. Apesar de que, segundo Grigoletto, Brito e Heredia (2014), a proposta do treinamento funcional ainda necessita de critérios para a aplicação do sistema de progressão, tendo como base os fundamentos do treinamento desportivo.

Portanto, trabalhar com esse tipo de treinamento requer de seus profissionais envolvidos a busca por desenvolver a capacidade de motivar seu aluno a não desistir da prática de exercícios físicos, cativando-o e proporcionando resultados significativos não somente para atingir seus objetivos e necessidades, mas proporcionar melhores condições de saúde e qualidade de vida.

0 termo "qualidade de vida" representa um contexto ligado a inúmeros campos do conhecimento da condição humana. Para Gonçalves e Vilarta (2004),qualidade de vida define-se pela maneira como as pessoas vivem, sentem e compreendem seu cotidiano, envolvendo, portanto, saúde, educação, transporte, moradia, trabalho e participação nas decisões que lhes dizem respeito.

No entanto, de acordo com a Organização Mundial da Saúde (OMS, 1995), essa temática trata também da percepção do indivíduo acerca de sua inserção na vida, no contexto da cultura e sistemas de valores nos quais ele vive, bem como sua relação com os seus objetivos, expectativas, padrões e preocupações desenvolvidas a partir do meio social, visto que toda sua construção envolve diretamente os aspectos ligados às condições e à maneira com que o sujeito vive.

Por outro lado, com o passar dos anos, essa qualidade de vida se tornou um dos principais objetivos a serem alcançados pelos sujeitos, principalmente por se tratar de um contexto que busca significativamente por melhores condições de saúde e bem-estar, seja a partir de novos hábitos alimentares, mudança de rotina e, sobretudo, pela prática orientada de exercícios físicos sob a orientação de um profissional qualificado, levandose em consideração suas inúmeras contribuições para com o desenvolvimento das perspectivas relacionadas ao bem-estar físico, psicológico e espiritual, além de aspectos sociais, econômicos e políticos.

Partindo desse princípio, o profissional de Educação Física deve, através de sua ação, planejar um processo em que os seus objetivos propostos estejam ligados à perspectiva de qualidade de vida, por meio de um programa de treinamento que possa viabilizar melhores condições de vida para seus praticantes, como por exemplo, o treinamento funcional.

Segundo Campos e Coraucci Neto (2008), o treinamento funcional é a mais recente maneira de se melhorar o condicionamento físico e a saúde em geral, com ênfase no aprimoramento da capacidade funcional do corpo humano, respeitando a individualidade biológica e permitindo que o corpo humano seja estimulado das diferentes formas possíveis.

Além disso, para Monteiro e Carneiro (2010), o treinamento funcional pode influenciar significativamente na melhora de diversos aspectos físicos, cognitivos, afetivos e biológicos, tais como: desenvolvimento da consciência sinestésica e controle corporal; melhora da postura; melhora do equilíbrio muscular; diminuição da incidência de lesões; melhora de estabilidade articular, principalmente da coluna vertebral; aumento da eficiência dos movimentos; 
melhora do equilíbrio estático e dinâmico; melhora da força e coordenação motora; melhora da resistência central cardiovascular e periférica-muscular; melhora da lateralidade corporal; melhora da flexibilidade e propriocepção.

Nesse tipo de treinamento, umas das principais características que devem ser consideradas para sua realização, além do interesse e motivação de seus praticantes, seria, então, a capacidade de explorar diversos tipos de exercícios que melhor proporcionam resultados positivos quanto aos objetivos esperados, bem como despertar um maior prazer e bem-estar de seus praticantes durante a sua realização.

Normman (2009), o treinamento funcional é ideal para ser aplicado em reabilitação de pacientes vítimas de sequelas, pois melhora o desempenho de praticantes de outras modalidades esportivas, previne lesões, oferece grande variação de exercícios e, com isso, é mais difícil de tornar-se monótono, além de ampliar as oportunidades no mercado de trabalho do Profissional de Educação Física.

Outra qualidade desse tipo de treinamento, segundo Ribeiro (2006), diz respeito à facilidade com que a performance pode ser acessível a qualquer pessoa, não somente a atletas, estimulando sempre o desenvolvimento de todas as capacidades físicas como força, velocidade, equilíbrio, coordenação, flexibilidade e resistência.

Dentro do treinamento funcional, todas essas etapas são de caráter progressivo, de tal forma que a sua essência deve ser respeitada para que o aluno possa desenvolver lentamente sua capacidade de controle neuromuscular, priorizando a realização de todos os movimentos com segurança e eficiência.

Quanto à prescrição das sessões de Treinamento Funcional, Evangelista (2011), salienta a forma de medir e quantificar a intensidade, por meio da percepção subjetiva de esforço, mediante o uso escalas que possibilitam a quantificação do esforço, como no caso da escala de OMNI-res, que consiste em uma linha que vai de zero a dez, onde, em cada exercício, o aluno lhe dá uma nota para o esforço que está sendo realizado, levando-se em consideração que não existe escala validada e específica para a percepção de esforço nas sessões desse tipo de treinamento, mas a percepção é dada de acordo com a interpretação de estímulos e feedback proporcionados pelo aluno.

Da mesma forma, vale ressaltar também que, de acordo com Normman (2009), o treinamento funcional não é caracterizado somente por um método de treino específico, mas se trata da combinação de vários movimentos, estimulando sempre diferentes grupos musculares. Utiliza-se, assim, diferentes materiais e aparelhos com a finalidade de propiciar a realização de diferentes tipos de ações motoras e movimentos semelhantes àsatividades básicas do cotidiano do sujeito.

No entanto, de acordo com Grigoletto, Brito e Heredia (2014), a proposta do treinamentofuncional ainda necessita de critérios específicos para a aplicação do sistema de progressão, tendo em vista que não existem, atualmente, estudos e comprovações que integram as metodologias tradicionais com as propostas desse tipo de treinamento, de maneira a possibilitar a construção de conceitos errôneos e fazendo com que esse tipo de treinamento não se faça tão eficaz como deveria ser.

Diante de todos os princípios elencados anteriormente, torna-se, então, importante ressaltar que o treinamento funcional representa uma nova metodologia de condicionamento que vem ganhando grande destaque no meio social, norteada pelas leis basais do treinamento e amparada cientificamente por meio de pesquisa e referências bibliográficas em todos os seus pontos principais, principalmente em relação à promoção de benefícios para a qualidade de vida. Partese da essência do treinamento funcional associado ao progresso dos aspectos neurológicos que comprometem a capacidade funcional do corpo humano através de treinos estimulantes que desafiam os vários componentes do sistema nervoso e, por isso, geram sua adaptação (D’ELIA; D’ELIA, 2005).

O profissional de Educação Física, no atual contexto social, vem ganhando grande destaque na atuação e desenvolvimento de ações motoras ligadas ao treinamento funcional, principalmente, por se tratar de uma nova metodologia de treinamento que atrai uma maior atenção daqueles que procuram por uma atividade diversificada que busca trabalhar todas as funções e capacidades do corpo.

Além disso, ele é responsável também por planejar, organizar e aplicar programas de treinamento especializados e direcionados para cada objetivo 
almejado por seus clientes, dentro das possibilidades discutidas pelas ciências do treinamento (LEITE, 2000). Ele se torna, então, o principal incentivador e motivador de seus clientes a adotarem novos hábitos saudáveis e a prática orientada de exercícios físicos.

No entanto, tratando-se de um programa de treinamento especialidade, deve-se considerar que, conforme salienta Monteiro (2006), sua prescrição depende totalmente da orientação de um especialista, sendo que o profissional de Educação Física é capacitado para exercer atividades por meio de intervenções, de avaliação, de prescrição e orientação de sessões de atividadesfísicas com fins educacionais, de treinamento, de prevenção de doenças e promoção da saúde. Considera-se que a sua formação profissional permite desenvolver um trabalho significativo e satisfatório voltado para os objetivos esperados por seus clientes/ alunos em relação à prática orientada.

Da mesma forma, torna-se papel do profissional de Educação Física avaliar as condições dos pacientes e elaborar um programa de atividades acessíveis ao grupo, possibilitando a realização de um trabalho permanente e eficaz, visando sempre à melhoria da qualidade de vida de seus clientes/alunos (ALVES, 2007).

Por outro lado, existe uma grande relação entre o tipo de atividade a ser desenvolvida e as metodologias utilizadas pelo profissional de Educação Física para a construção de seu planejamento. No treinamento funcional, por exemplo, por se tratar de uma atividade que possibilita uma maior diversidade de movimentos e exercícios, o profissional envolvido deve promover um programa de treinamento criativo, prazeroso e que possibilita despertar um maior interesse e desejo de seus clientes pela prática esportiva como benefício para sua qualidade de vida.

Para Weineck (1999), tratando-se do treinamento, o profissional de Educação Física responsável pela orientação do aluno deve possuir o conhecimento básico dos princípios do treinamento esportivo, pois estes determinam o programa e o método a ser utilizado, bem como a organização dos mesmos, tendo em vista queessesprincípios constituemparâmetrosimportantes tanto para o treinador como para o aluno.

Além disso, trazendo para a perspectiva do treinamento funcional, o profissional de Educação
Física deve, antes de tudo, entender que cada aluno possui uma individualidade biológica associada à necessidade e objetivo próprio. Logo, cada exercício deve ser pensado e planejado para atender todas essas particularidades dentro de um programa especializado.

Isto porque, de acordo com Franco (2008), o Treinamento Funcional é um método de treinamento físicoquetemporbaseodesenvolvimentoneuromuscular integrado, cujo objetivo prioritário é tornar o corpo humano uma máquina de locomoção mais eficiente, melhorando seu desempenho, além de contribuir para a prevenção de lesões e estimular um trabalho músculoesquelético de forma ampla e conjunta, ao invés de segmentada e isolada.

Sabe-se que existem alguns princípios importantes que devem ser considerados em se tratando de um programa de treinamento funcional, assim, como é salientado por Monteiro e Evangelista (2011), uma metodologia de treinamento só pode ser considerada como funcional se o aluno apresentar, dentre outras, as seguintes características: a sustentação do centro de gravidade, suporte para a postura durante a execução dos exercícios; desenvolvimento das capacidades motoras; padrão de movimento comparável a reflexos; compatibilidade com um programa motor generalizado, movimentos transferidos para as atividades diárias; isolamento para integração, treinando musculaturas isoladamente para colaborar com a realização do movimento funcional.

No treinamento funcional, como nos demais treinamento físico, o conhecer seu aluno representa uma tarefa de suma importância para o profissional de Educação Física saber como planejar corretamente os exercícios a serem realizados nesse tipo de programa. Para Silva (2010), torna-se importante entender que cada aluno possui sua individualidade, uma vez que a necessidade e o objetivo de cada um são diferentes.

Portanto, cabe aqui ressaltar que a presença do profissional de Educação Física torna-se de suma importância para o desenvolvimento de um programa de treinamento especializado, levando em consideração uma orientação adequada para o planejamento e prescrição dos exercícios a serem realizados, seja para o treinamento funcional ou qualquer outro tipo de treinamento físico. Esses profissionais também são responsáveis por preparar o indivíduo para ter maior 
disposição e energia para realizar as atividades diárias, bem como promover melhores condições de saúde, qualidade de vida e bem-estar.

\section{MATERIAIS E MÉTODOS}

O presente estudo caracterizou-se como uma pesquisa de campo com abordagem quantitativa com a proposta deanalisar os benefícios proporcionados pelo treinamento funcional para a promoção de qualidade de vida em moradores da área rural de Paripiranga (BA).

Aamostra foi constituída por 30 participantes (15 participantes considerados ativos e 15 participantes considerados insuficientemente ativos), com idade entre 25 a 50 anos, moradores da área rural da comunidade Sabão, município de Paripiranga (BA), Nordeste, Brasil.

Os critérios de inclusãoestabelecidos foram: ter idade acima de 18 anos, participar voluntariamente da pesquisa, assinandoum Termo de Consentimento Livre e Esclarecido e responder aos questionários IPAQ e o WHOQOL-Bref.Foi considerado como critérios de exclusãoaqueles que nãoatendessem atais pressupostos estabelecidos anteriormente. 0 trabalhofoi submetido à apreciação e aprovado pelo Comitê de Ética e PesquisaEnvolvendo Seres Humanos do Centro Universitário AGES, Paripiranga, Bahia.

A amostra foi constituída por doisgrupos: Grupo de Treinamento Funcional (GTF, n=15) e Grupo Controle ( GC, n=15). O GTF foi composto por indivíduos ativos,praticantes deum programa de treinamento funcionalpor umperíodo de6 meses, com frequência de 3 vezes por semana e duração de 50 minutos por sessão. Já o GC foi constituído por indivíduos insuficientemente ativos que não receberam intervenção.

\section{PROCEDIMENTOS}

Inicialmente, todos as participantes responderam ao Questionário Internacional de Atividades Físicas (IPAQ) para classificaçãoem ativo ouinsuficientemente ativo. 0 ponto de corte para a classificação de ativo foi de $150 \mathrm{~min} /$ semana. O IPAQ constitui um instrumento simples e de fácil preenchimento e serve como ferramenta de verificação dos níveis de atividade física (BARROS; NAHAS, 2003).

No final das intervenções foi aplicado o WHOQOLbrefque é um instrumento curto e de rápida aplicação, contendo26questõesobjetivas, sendo,pois, considerado um instrumento transcultural que valoriza a percepção individual da pessoa, tendo como principal finalidade avaliar qualidade de vida em diversos grupos e situações (VESPASIANO; DIAS; CORREIA, 2012).

\section{Protocolo de Treinamento Funcional}

Os sujeitos do grupo de treinamento funcional (GTF) realizaram sessões de treinamento funcional, por um período de 6 meses, com frequência de 3 vezes por semana e com duração de 50 minutos em um salão, lotado na comunidade Sabão, município de Paripiranga (BA).

Asessão foi dividida em parte inicial com aquecimento (10 min). Parte principal com circuitos em estações (30 min)e parte final com alongamentos (10 min). 0 programa de treinamento funcional consistiu emexercícios intervalados como corridas curtas, circuito envolvendo series curtas de exercícios (1 a 3 repetições de 15 a 20 vezes) livres e com pesos, saltos, equilíbrios, flexibilidade, abdominais, resistências (COELHO NETTO e APTEKMANN, 2016).

Para a análise dos dados, ocálculo dos escores e estatística descritiva do WHOQOL-breffoi realizadoatravésdoSoftware MicrosoftExcel(PEDROSO, et al, 2010).

\section{RESULTADOS E DISCUSSÃO}

A amostra foi composta por 12 (40\%) participantes do sexo masculino, correspondendo a e 18 (60\%) do sexo feminino. Sua distribuição foi constituída em 15 (50\%) participantes considerados ativos, e 15 (50\%) participantes considerados insuficientemente ativos, mediante análise feita em relação ao nível de atividade física em que estes se encontram de acordo com os dados obtidos pelo questionário IPAQ. 
Tabela 1. Estatística descritiva dos participantes ativos e insuficientemente ativos (IPAQ).

\begin{tabular}{|c|c|c|}
\hline Participantes & $\mathrm{n}^{\mathrm{o}}$ & $\%$ \\
\hline \multicolumn{3}{|l|}{ Sexo } \\
\hline Masculino & 12 & $40 \%$ \\
\hline Feminino & 18 & $60 \%$ \\
\hline \multicolumn{3}{|l|}{ Nível de Atividade Física } \\
\hline Ativos & 15 & $50 \%$ \\
\hline Insuficientemente ativos & 15 & $50 \%$ \\
\hline
\end{tabular}

Fonte: Dados do autor (2019)

Gráfico 1. Escala de domínios da qualidade de vida dos participantes ativos (WHOQOL-bref).

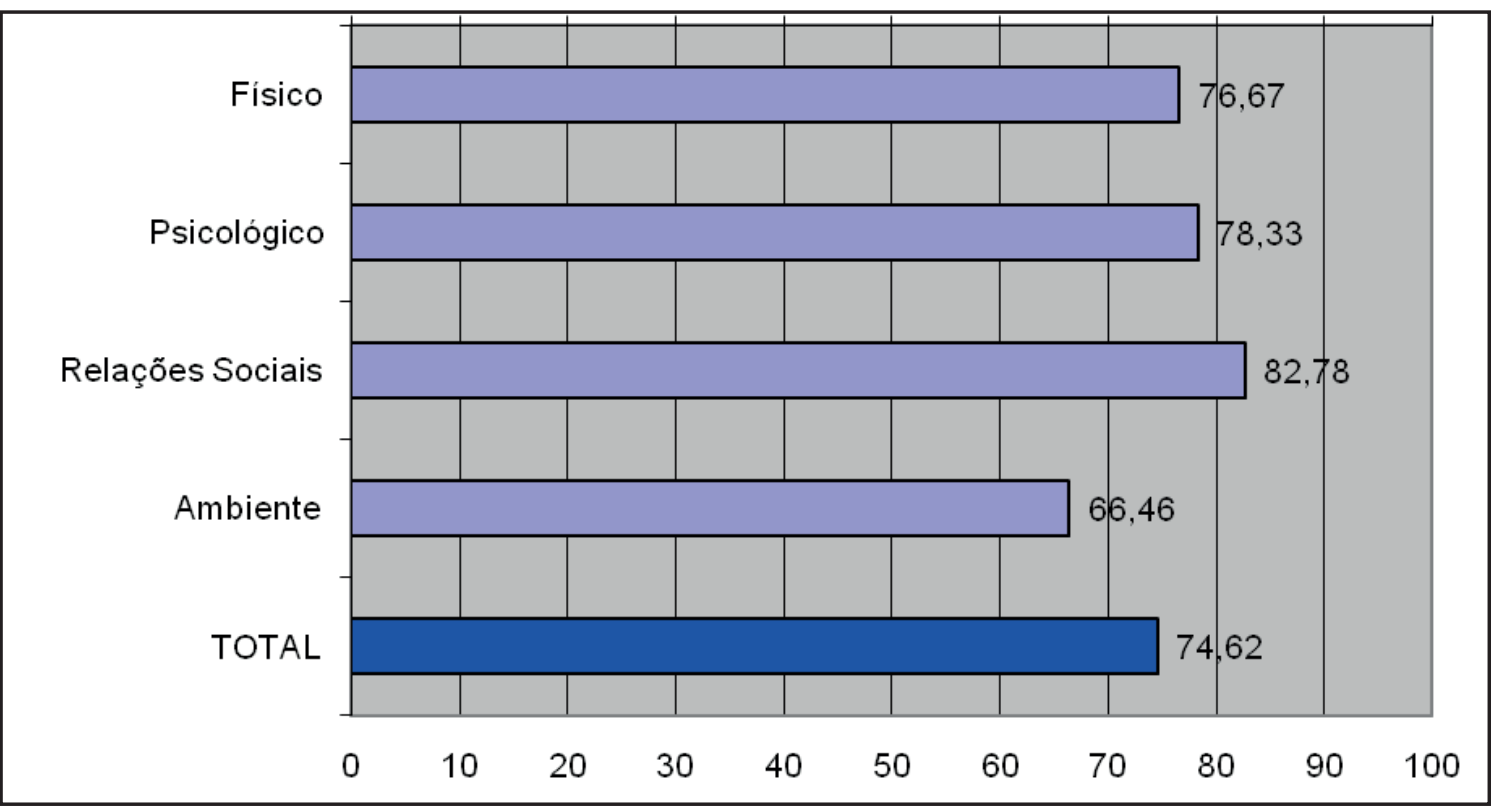

Fonte: Dados dos autores (2019)

0 Gráfico 1, apresenta os dados referentes à escala de domínio da qualidade de vida dos participantes considerados ativos, trazendo como enfoque principal a relação dos domínios físicos, psicológicos, relações sociais e ambiente associados aos benefícios proporcionados pela prática regular de um programa de treinamento funcional com o desenvolvimento de cada domínio.

Em relação ao domínio físico e ao psicológico, os dados apresentaram um valor satisfatório de 76,67\%, e 78,33\%, respectivamente. No domínio de relações sociais verificou-se $82,78 \%$, levando em consideração que, quanto mais os valores aproximam-se da porcentagem de $100 \%$, melhores os resultados para a análise da qualidade de vida dos participantes.
Para Campos e Coraucci Neto (2008), o treinamento funcional contribui significativamente na melhoria dos aspectos neurológicos que afetam diretamente a capacidade funcional do corpo humano, utilizando exercícios que desafiem os diferentes componentes do sistemanervosoeque,assim, estimulamodesenvolvimento tantos dos aspectos físicos como dos aspectos psicológicos necessários para sua motivação e adaptação.

Logo, as melhorias dos aspectos físicos, emocionais e psicológicos estão diretamente ligadas aos domínios da qualidade de vida, visto que o exercício físico em um sentido amplo se torna um aspecto influenciador para a melhoria de demais domínios da vida das pessoas (COSTA; DUARTE, 2006). 
O Gráfico 2 ressalta a escala de domínios de qualidade de vida dos participantes considerados insuficientemente ativos, trazendo em sua construção o mesmo enfoque dado aos domínios físicos, psicológicos, relações sociais e ambiente, associando a sua rotina e atividades diárias com o desenvolvimento de cada domínio.

Gráfico 2. Escala de domínios de qualidade de vida dos participantes insuficientemente ativos (WHOQOL-bref)

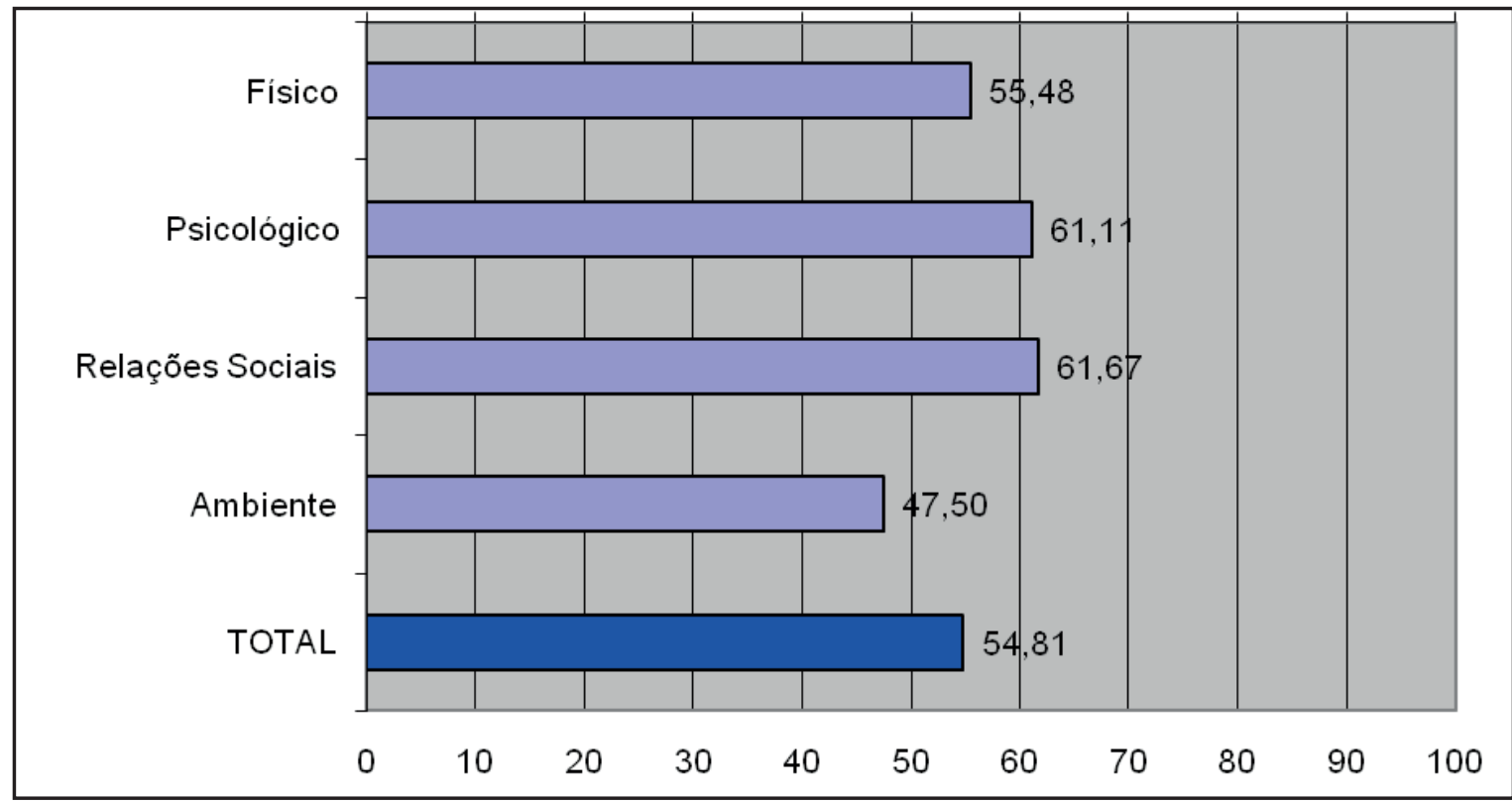

Fonte: Dados dos autores (2019)

No primeiro domínio direcionado para o aspecto físico, os dados coletados constatouo valor de 55,48\%; enquanto que, nos aspectos psicológicos, apresentou-se um valor de 61,11\%; bem como os aspectos ligados ao domínio das relações sociais, que também apresentou um valor semelhante de 61,67\%; e, por fim, o domínio do ambiente com apenas 47,50\%, um pouco abaixo da média esperada para análise ideal da qualidade de vida dos participantes envolvidos.

Diante desse contexto e conforme é salientado por Sallis e Owen (1997), o processo de saúde e qualidade de vida é influenciado por múltiplas facetas que vão desde os aspectos físicos, sociais e do ambiente. No entanto, as condições do ambiente podem dificultar a elaboração de um estilo de vida ativo, que permita que o sujeito possa desempenhar suas atividades diárias com maior disposição, bem como melhorar o desenvolvimento de seus aspectos físicos, psicológicos, afetivos e sociais.

Logo, considera-se que os dados obtidos no Gráfico 2 , relacionados aos participantes considerados insuficientemente ativos, apresentam-se bem abaixo dos resultados que foram destacados no gráfico 1 referente aos participantes ativos. Levando em consideração que a falta de uma prática regular de exercícios físicos pode ter influenciado negativamente para que todos os dados estivessem abaixo do esperado para avaliar a qualidade de vida.

Isto porque um estilo de vida ativo pode ser resultante da prática de exercícios físicos que poderá contribuir para uma boa condição física, psicológica e social, sendo também considerado um dos importantes fatores para a prevenção e o tratamento de doenças e para a manutenção da saúde, bem como um instrumento precioso para a melhoria de qualidade de vida das pessoas (ARAUJO; ARAUJO, 2000).

No gráfico 3 , apresenta-se os dados referentes à escala de variáveis de qualidade de vida dosparticipantes ativos. Dentre eles, vale destacar os dados referentes à dor e desconforto (18,33\%); dependência de medicação $(13,33 \%)$; sentimentos negativos $(15 \%)$; recursos financeiros (55\%) e cuidados de saúdes (43,33\%). 
Gráfico 3. Escala de variáveis de qualidade de vida dos participantes ativos (WHOQOL-bref)

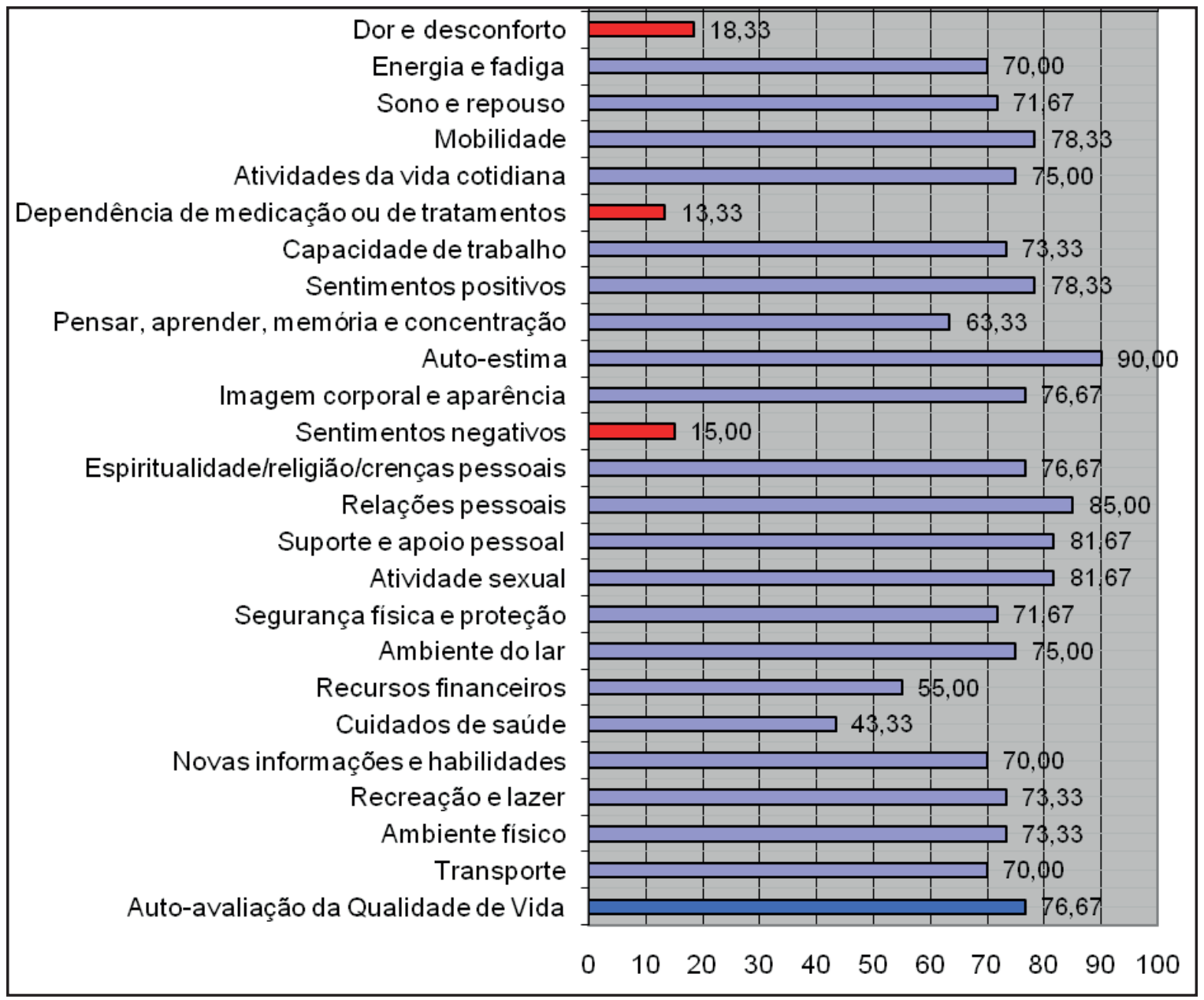

Fonte: Dados dos autores (2019)

Os dados constituem os menores valores dentre todas as variáveis apresentadas, considerando que os pacientes ativos demonstraram dados positivos quanto a menores índices de dor e desconforto durante a sua rotina diária, bem como baixa dependência do uso de medicamentos e pouca incidência de sentimentos negativos. Considerou-se também que a prática de exercícios físicos regulares, assim como o treinamento funcional tem influenciado para a promoção de resultados significativos para com essas variáveis salientadas.

Para Cossenza e Carvalho (1997), um programa de exercício físico pode ser um meio alternativo excelente para descarregar ou liberar tensões, emoções e frustrações acumuladas pelas pressões e exigências da vida moderna. Ele é, também, uma importante ferramenta para desenvolver a capacidade de lidar com situações de estresse, ansiedade, além de diminuir a incidência de casos de depressão e tensão.

Com relação ao uso de medicamentos e incidência da dor e desconforto, Scoss eSalviano (2013) salientam que o treinamento funcional desenvolve melhorias fisiológicas, psicológicas, morfológicas, bioquímicas e funcionais, quesão desenvolvidas através de adaptações produzidas por este treinamento. Além de produzir um aumento significativo das variáveis relacionadas à aptidão física e à capacidade funcional em realizar suas atividades diárias, com um aumento da força muscular, potência, resistência, flexibilidade, equilíbrio, coordenação motora, condicionamento cardiovascular, dentre outras, diminuindo assim o risco de acidentes no cotidiano, uso de medicamentos constantes e a existência de dores musculares persistentes.

Da mesma forma, para Costa e Duarte (2002), o exercício físico pode diminuir a prevalência da dor e 
melhorar a função de condições relacionadas à dor crônica, sendo um tratamento eficaz para várias doenças que apresentam fortes dores como a fibromialgia, dor de garganta crônica, osteoartrite, artrite reumatóide e dor lombar crônica.

Por outro lado, em relação aos dados referentes aos recursos financeiros e cuidados com a saúde, pode-se perceber que essas duas variáveis apresentam uma pequena queda dos índices esperados para uma boa qualidade de vida, comparando-se com as demais variáveis destacadas pelo Gráfico 3.

Partiu-se do pressuposto de que a falta de recursos financeiros apresenta grande associação com os cuidados com a saúde, na medida em que uma renda familiar baixa pode impossibilitar que uma família tenha todo o acesso aos meios de cuidados com a saúde. Assim, é dever das políticas públicas darem todo suporte e acompanhamento às famílias na promoção e prevenção da saúde, bem como na promoção de uma melhor qualidade de vida.

Conforme salienta Leff (2002), as condições de saúde dos seres humanos constituem elemento primordial para a promoção da qualidade de vida, levando-se em consideração também as noções de bemestar, nível de vida, condições de existência e estilos de vida, valores culturais e identidades étnicas, fazendo uma relação direta entre o ambiente e a satisfação das necessidades básicas.

Por outro lado, ainda no Gráfico 3, pode-se ressaltar a prevalência de alguns dados positivos que estão acima da média esperada, bem como estão associados aos benefícios proporcionados pela prática regular de exercícios físicos, tais como: energia e fadiga (70\%), sono e repouso $(71,67 \%)$, mobilidade $(78,33 \%)$, sentimentos positivos (78,33\%), autoestima (90\%), relações pessoais (85\%), atividade sexual $(81,67 \%)$ e recreação e lazer $(73,33 \%)$, totalizando uma média geral de 76,67\%.

Partindo do princípio de que um estilo de vida ativo proporciona inúmeros benefícios para todas as variáveis discutidas em relação à qualidade de vida do sujeito, a prática regular de um programa de treinamento funcional pode apresenta grandes benefícios para todos os sistemas do corpo.

Isto porque, conforme é salientado por Alfieri, Teodori e Montebelo (2004), além do treinamento funcional apresentar melhorias na composição corporal dos sujeitos praticantes, ele também colabora para a redução de problemas emocionais e sociais atribuídos à incapacidade,sendocapazdemelhorarodesenvolvimento das capacidades de equilíbrio, coordenação e agilidade, beneficiando tanto as condições de saúde quanto promovendo a independência desse público.

Logo, a qualidade de vida deixou de representar apenas uma vida sem doenças, mas, acima de tudo, está ligada à busca pela felicidade e satisfação pessoal diante todos os aspectos da vida de forma integrada (COSTA; DUARTE, 2006).

Contudo, por meio do presente estudo, pode-se ressaltar que os indivíduos considerados ativos e que participam de programas de treinamento funcional possuem uma melhor qualidade de vida mediante análise feita, se comparados aos indivíduos insuficientemente ativos que não praticam quaisquer exercícios físicos. Essa diferença se dá pelo fato de os participantes destes programas de treinamento convivem mais tempo em grupo, sentem-se capacitados e independentes, facilitando a participação dos mesmos na sociedade e possibilitando a realização das atividades cotidianas com maior satisfação e disposição (ARAGÃO; DANTAS; DANTAS; 2002).

No Gráfico 4 apresentas os dados referentes às variáveis de qualidade de vida dos participantes considerados insuficientemente ativos. Cabe-se aqui destacar as variáveis relacionadas à dor e ao desconforto (35\%), dependência de medicação (35\%), sentimento negativo (35\%), recursos financeiros $(36,67 \%)$ e cuidados com a saúde (25\%), no qual obtiveram os menores resultados quanto à análise da qualidade de vida, em comparação comas demais variáveis enfatizadas.

Os dados ressaltam que os participantes insuficientemente ativos possuem maiores queixas em relação à prevalência de dores e desconforto durante o desenvolvimento de suas atividades diárias, bem como possuemumamaiordependênciadousodemedicamentos constantese, também, apresentam sentimentosnegativos com maior frequência em comparação aos dados coletados para os participantes ativos.

Para Gravina, Grespan e Borges (2007), as pessoas consideradas insuficientemente ativas apresentam maior probabilidade de desenvolver diferentes tipos de doenças crônicas, além de estarem propícios também ao uso de medicamentos constantes como forma de tratamento. 
Gráfico 4. Escala de variáveis de qualidade de vida dos participantes insuficientemente ativos (WHOQOL-bref).

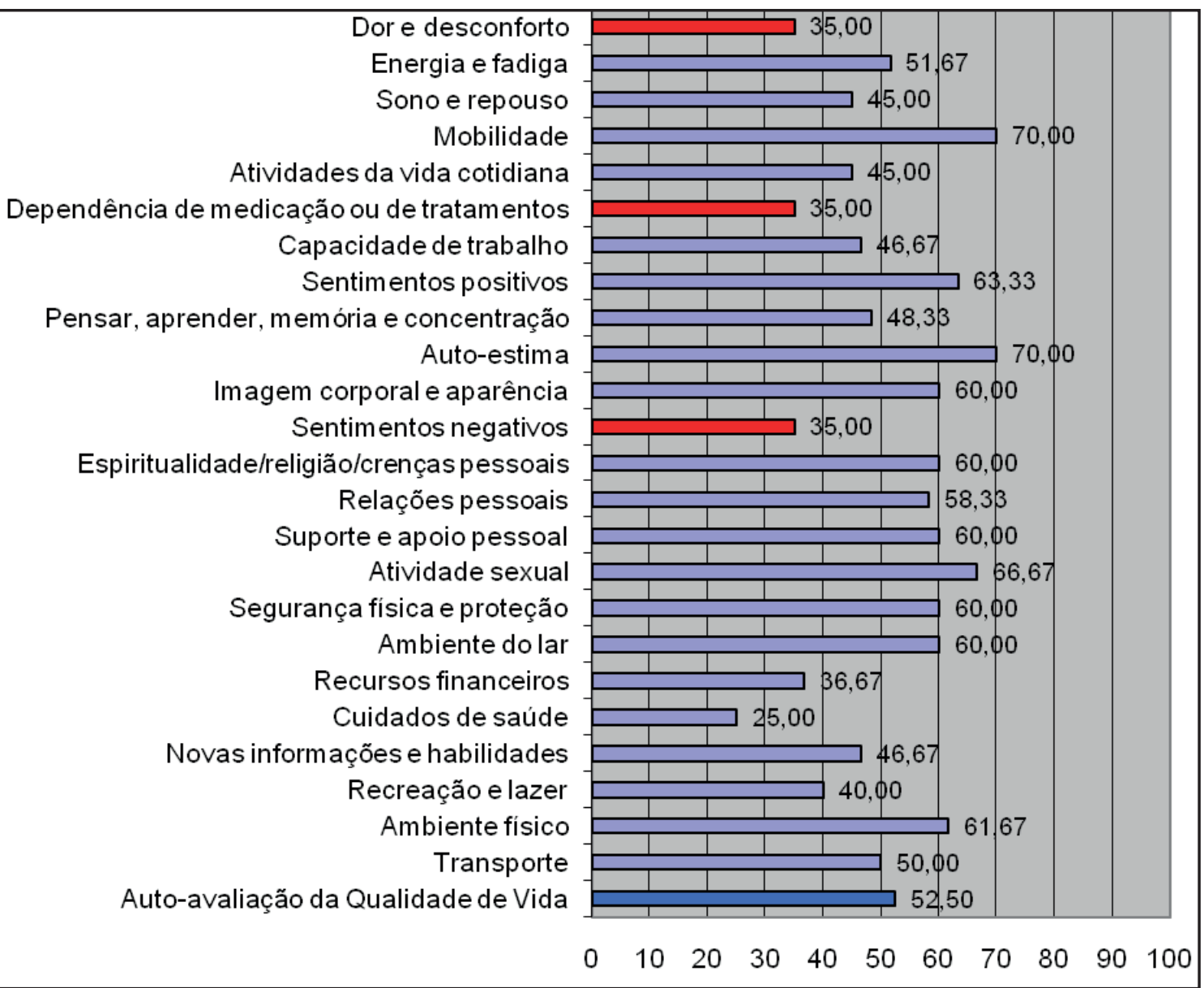

Fonte: Dados dos autores (2019)

Em relação às variáveis relacionadas aos recursos financeiros e cuidados com a saúde,Fabiancic (2016) ressalta que as pessoas consideradas insuficientemente ativas podem diminuir seu potencial de ganhos salariais na vida profissional, comprometendo sua produtividade e renda familiar e aumentando os níveis de ausências no trabalho devido aos períodos de afastamento por licença médica, gerando mais gastos com tratamento para os empregadores e para o sistema de saúde. Dessa forma, além dos benefícios sociais, a prática regular de exercícios físicos também pode ser vista como uma forma efetiva de medicina preventiva, tanto para os indivíduos quanto para os sistemas governamentais e empresas.

Por outro lado, o Gráfico 4 apresenta também outras variáveis que são consideradas importância para análise da qualidade de vida, como: mobilidade (70\%), sentimentos positivos $(63,33 \%)$, autoestima (70\%), atividade sexual $(66,67 \%)$ e ambiente físico $(61,67 \%)$, totalizando uma média geral de apenas 52,50\%. Considerando que a maior parte dos resultados apresentados se encontra entre a média idealiza, porém um pouco abaixo dos dados apresentados no Gráfico 3, comparando-se com os participantes ativos.

Isto porque, apesar dos resultados apresentarem uma boa relação de uma parte das variáveis analisadas, deve-se preconizar que os sujeitos que participam do programa de treinamento funcional apresentaram níveis elevados, estando em sua maioria acima da média idealizada para a análise das variáveis da qualidade de vida, levando em consideração os benefícios proporcionados pela prática regular de exercícios físicos.

Segundo Ambrose e Golightly (2015), a prática regular de exercícios físicos proporciona inúmeros benefícios para a saúde geral, ajuda no controle dos 
riscos de doenças crônicas como doenças cardiovasculares, diabetes tipo 2 e obesidade. Nesse contexto, o exercício desencadeia a liberação de betaendorfina da hipófise (perifericamente) e do hipotálamo (central) que, por sua vez, proporciona efeitos analgésicos através da ativação de receptores opióide perifericamente e centralmente. Os programas de atividade física podem proporcionar a oportunidade de alargar as relações sociais, estimulando novas amizades e gerando efeitos saudáveis no nível cognitivo e na qualidade de vida.

Da mesma forma, neste estudo foram encontradas diferenças significativas em relação aos dados obtidos para os participantes ativos e os participantes insuficientemente ativos, considerando, principalmente, a relação do programa de treinamento funcional como sendo um importante fator para a promoção de inúmeros benefícios ligados à qualidade de vida, tendo em vista que é possível notar que os participantes ativos apresentaram índices de qualidade de vida acima da média.

\section{CONCLUSÃO}

Os resultados revelaram que os indivíduos considerados ativos apresentaram aumento dos índices das variáveis ligadas às melhorias da qualidade de vida, tais como: melhor disposição para o sono e repouso; maior mobilidade; maior disposição para o desenvolvimento das atividades da vida cotidiana; menor aderência ao uso constante de medicamentos; maior incidência de sentimentos positivos; aumento elevado da autoestima e aspectos relacionados à imagem corporal e aparência, melhora significativa no desenvolvimento das relações pessoais; maior disposição para as atividades sexuais; dentre outras variáveis analisadas.

Em relação aos participantes insuficientemente ativos, verificou-se que a falta da prática regular de exercícios físicos pode interferir diretamente na melhoria de alguns aspectos relacionados à qualidade de vida, tais como: presença constante de dor e desconforto durante a realização das atividades diárias; prevalência de sentimentos negativos; dificuldades relacionadas ao sono e repouso; menor disposição para o desenvolvimento das atividades da vida cotidiana; menor disponibilidade para manutenção dos cuidados voltados para a saúde; além de outras variáveis apresentadas.

Em suma, torna-se importante ressaltar que a prática regular de um programa de treinamento funcional poderá contribuir satisfatoriamente para o aumento das variáveis relacionadas à qualidade de vida, bem como possibilitar melhorias para a capacidade funcional do ser humano e aspectos relacionados à autoestima, autonomia e condições favoráveis para o desenvolvimento das relações sociais.

Destaca-se a importância do profissional de Educação Física na prescrição e orientação de exercícios físicos, como o treinamento funcional, a fim de contribuir significativamente para a melhoria da qualidade de vida da população. 


\section{REFERÊNCIAS}

ALFIERI,F.M.;TEODORI.R.M.;MONTEBELO,M.I.L. Mobilidade funcional de idosos submetidos a intervenção fisioterapêutica. SaúdeRev: Piracicaba. 2004.

ALVES, C. A Importância do Profissional de Educação Física no Tratamento do Indivíduo com Diabetes Tipo II. 2007.

AMBROSE, K.R.; GOLIGHTLY, Y.M. Physical exercise as nonpharmacological treatment of chronic pain: Why and when. Best Pract Res Clin Rheumatol 2015

AMERICAN COLLEGE OF SPORTS MEDICINE (ACM). American College of Sports Medicine position stand. Progression models in resistance training for healthy adults. Med Sci Sports Exercise, 2009.

ARAGÃO, J.C.B; DANTAS, E.; DANTAS, B. Resistência Muscular Localizada e Autonomia. Rev. Fit. e Per. Jour. 2002.

BARROS, M.V.G; NAHAS, M.V. Medidas da Atividade Física. Teoria da Aplicação em Diversos Grupos Populacionais. Londrina, Midiograf, 2003.

BOSSI, L. C. Treinamento funcional na musculação. São Paulo: Phorte, 2011.

CAMPOS, M. A; CORAUCCI NETO, B. Treinamento Funcional Resistido: Para Melhoria da Capacidade Funcional e Reabilitação de Lesões Musculoesqueléticas. Rio de Janeiro, RJ: Revinter, Reimpressão, 2008.

COELHO NETTO, JS, APTEKMANN, NP. Efeitos do treinamento funcional sobre a composição corporal: um estudo em alunos fisicamente ativos de academia. RevBrasFisiolExerc. 2016; 15(2):68-76.

COSSENZA,C. E.; CARVALHO, N. Personal training para grupos especiais. Rio de Janeiro: Sprint, 1997.

COSTA, A.M; DUARTE, E. Atividade física e a relação com a qualidade de vida, de pessoas com sequelas de acidente vascular cerebral isquêmico (AVCI). RevBrasCienMov, 2002.

COSTA,A.M; DUARTE, E. Atividade Física, Saúde e a Qualidade de Vida das Pessoas com Deficiência. In: Rodrigues, D. (Org.). Atividade Motora Adaptada - A Alegria do Corpo. 1 ed. São Paulo: Artes Médicas; 2006.

D’ELIA, R.; D'ELIA, L. Treinamento funcional: $7^{\circ}$ treinamento de professores e instrutores. São Paulo: SESC - Serviço Social do Comércio, 2005.

EVANGELISTA, A.L. Prescrição do treinamento funcional: aspectos a serem considerados. 2011.
FABIANCIC, N. Documento Referencial: Projeto Escolas e Comunidades Ativas. Rio de Janeiro, 2016.

FARIAS, M. Treinamento Funcional, bodycontrolstudium. wordpress. 2015.

FRANCO, A. Essência do treinamento funcional. 2008.

GELATTI, P. 0 gladiador do futuro. Combat Sport. São Paulo, n. 46, p. 12-14, 2009.

GOLDENBERG, L.; TWIST, P. Strength ball training: 69 exercises using Swiss balls and medicine balls. United States of America. Human Kinetics. 2002.

GONÇALVES, A. Em busca do diálogo do controle social sobre o estilo de vida. In: VILARTA, Roberto (org.) Qualidade de Vida 137 e políticas públicas: saúde, lazer e atividade física. Campinas, IPES, 2004

GRAVINA, C.F.; GRESPAN S.M.; BORGES, J.L. Tratamento não medicamentoso da hipertensão em idoso. 2007.

GRIGOLETTO, M.E.; BRITO, C.J.; HEREDIA, J.R. Treinamento funcional: funcional para que e para quem? Rev. Bras. Cineantropometria desempenho humano. 2014.

LEFF, E. Saber ambiental: sustentabilidade, racionalidade, complexidade, poder. Petrópolis: Vozes, 2002.

LEITE, P. F. Aptidão Física, Esporte e Saúde. 3. Ed. São Paulo: Robe, 2000.

MICHAELIS, A. Dicionário de língua portuguesa. São Paulo, 2009.

MONTEIRO, A.G.; EVAGELISTA, A.L. Treinamento Funcional: Uma abordagem prática. São Paulo: Phorte, 2010.

MONTEIRO, A; CARNEIRO.T; O que é Treinamento Funcional? São Paulo, SP: Phorte, 2010.

Monteiro, L. Z. Perfil da Atuação do Profissional de Educação Física Junto aos Portadores de Diabetes Mellitus nas Academias de Ginástica de Fortaleza. Dissertação de mestrado em Educação em Saúde - UNIFOR. Fortaleza, 2006.

NAHAS, M. V. Atividade Física, saúde e qualidade de vida: conceitos e sugestões para um estilo de vida ativo. Londrina: Midiograf, 2001.

NORMMAN, T. Treinamento funcional: o novo divisor de águas. 2009

ORGANIZAÇÃO MUNDIAL DE SAÚDE (OMS). The World Health Organization Quality of Life Assessment(WHOQOL): position paper from the World Health Organization. Social scienceand medicine. v.41, n.10, 1995 
PEDROSO, B. etal. Cálculo dos escores e estatística descritiva do WHOQOL-bref através do Microsoft Excel. Revista Brasileira de Qualidade de Vida, Ponta Grossa, v. 2, n. 1, p. 3136, jan./jun. 2010.

RIBEIRO, A. P. F. A eficiência do Treinamento Funcional Resistido. Centro de Pós-graduação e pesquisa da UNIFMU, CENTRO UNIVERSITÁRIO, São Paulo, 2006.

SALLIS, J. F.; OWEN, N. Ecological Models. In K. Glanz, F.M Lewis, e B.K. Rimer (Orgs). Health Behavior and Health Education: Theory, Research and Pratice. (pp 403-424). San Francisco, CA: Jossey-Bass. 1997.

SCOSS, D. M.; SALVIANO, K. N. Os benefícios do treinamento de força sobre alterações dos sistemas fisiológicos associados à saúde na terceira idade. FiepBulletin, v.83, SpecialEdition, 2013.
SILVA, B. R. V. A ciência do corpo perfeito. São Paulo: Editora universo dos livros, 2010.

SILVA, L.X.N. Revisão da Literatura acerca do Treinamento Funcional Resistido e seus Aspectos Motivacionais em Alunos de Personal Trainning. Trabalho de conclusão de curso apresentado ao curso de Educação Física da Universidade Federal do Rio Grande do Sul, Porto Alegre, 2011.

VESPASIANO, B. S.; DIAS, R.; CORREIA, D. A. A utilização do Questionário Internacional de Atividade Física (IPAQ) como ferramenta diagnóstica do nível de aptidão física: uma revisão no Brasil. Saúde Rev., Piracicaba, v. 12, n. 32, p.4954, set.-dez. 2012.

WEINECK, J. Treinamento ideal. São Paulo: Editora Manole, 1999. 